\title{
A REASSESSMENT OF THE HEAT TRANSPORT BY VARIABLE VISCOSITY CONVECTION WITH PLATES AND LIDS
}

\author{
Michael Gurnis
}

\author{
Department of Geological Sciences, The University of Michigan, Ann Arbor
}

\begin{abstract}
The heat transport by a viscous fluid with temperature dependent viscosity has been studied numerically. As opposed to previous models, the top surface of the fluid clearly defines a tectonic plate with horizontally uniform velocity and subduction. Past studies failed to incorporate plates resulting in inefficient heat transport. With tectonic plates, the heat transport is as efficient as Rayleigh-Benard convection with constant viscosity; there is a strong buffering between internal temperature and heat loss. Past studies of parameterized convection which incorporated parameters indicative of a strong buffering between internal temperature and total heat output still provide the most physically plausible representation of the Earth's thermal evolution.
\end{abstract}

The temperature dependence of silicate rheology has long been thought to imply a strong buffering between terrestrial heat loss and internal temperature. This process operates such that the viscosity of a warming planet decreases and leads to increased heat advection and heat output. This principle of planetary evolution has been brought into serious doubt as numerical models of convection with temperature dependent viscosity apparently failed to exhibit such behavior [Christensen, 1984, 1985]. The present paper critically examines this discrepancy between theory and fluid dynamic models.

This principle of temperature buffering, along with a relation between heat loss (Nusselt number) and Rayleigh number, has been exploited to formulate thermal history models of the Earth's mantle. These models are in general accord: predicting, for example, that during the Archean the mantle heat flux was $\sim 4$ times higher than present values, while the internal temperature was only about $100^{\circ} \mathrm{C}$ higher [Davies,1980; Jackson and Pollack,1984; Schubert et al.,1980; Turcotte et al., 1979]. Such thermal history models assume a power law relation between heat loss and Rayleigh number

$$
\mathrm{Nu}=\mathrm{kRa}^{\beta}
$$

where $\mathrm{Nu}$ is the Nusselt number, $\mathrm{Nu}=\mathrm{q} / \mathrm{q}_{\mathrm{c}}$ where $\mathrm{q}$ is the total heat transferred out of the system, and $q_{c}$ is the heat which would be transported by conduction alone assuming no convection with a temperature difference $\Delta T, k$ and $\beta$ are constants, and $\mathrm{Ra}$ is the Rayleigh number. For bottom heated convection (i.e. Rayleigh-Benard convection), $\mathrm{Ra}$ is usually defined as

$$
\mathrm{Ra}_{1 / 2}=\frac{g \rho \alpha \Delta \mathrm{TD}^{3}}{\mathrm{k \eta}\left(\mathrm{T}_{1 / 2}\right)}
$$

where $T_{1 / 2}$ is the mean temperature, $T_{b}-\Delta T / 2, T_{b}$ is the bottom temperature, $\alpha$ is the coefficient of thermal expansion, $g$ is the acceleration of gravity, $\rho$ is the density, D is the layer

Copyright 1989 by the American Geophysical Union.

Paper number $89 \mathrm{GL} 00018$.

0094-8276/89/89GL-00018\$03.00 depth, $k$ is the thermal conductivity, and $\eta\left(T_{1 / 2}\right)$ is the viscosity at the mean temperature.

The thermal history models cited above employed a $\beta \approx 1 / 3$ which is valid for convection in a constant viscosity fluid and has been derived from boundary layer theory [Turcotte and Schubert, 1982] and verified numerically [Schubert and Anderson, 1985]. Recently, Christensen [1984, 1985] has made an in depth study of the relation between $\mathrm{Nu}$ and $\mathrm{Ra}$ for a fluid with a strong temperature dependence of viscosity and argues that the form of $(I)$ is invalid. Christensen argues that $\beta$ is dependent on $\operatorname{Ra}$ and that at large $\operatorname{Ra}\left(>10^{7}\right) \beta$ actually vanishes (i.e. $\beta=0$ ). He argues that $\beta \approx 1 / 10$ is the value appropriate for the Earth's mantle over most of its history. If this were true, then the process of thermal buffering in the Earth's interior is muted and a very different terrestrial thermal/tectonic evolution emerges in which plate velocities and heat flux remain virtually unchanged over the last $\approx 4 \mathrm{Ga}$. However, the mantle would have a much higher internal temperature [Christensen, 1985].

In the next section, the models of Christensen [1984, 1985] are critically examined. It is argued that these models fail to incorporate subduction, the principal means by which the Earth's mantle is presently cooled. It is shown that these models generate seismic velocity variations beneath downwellings which are too small compared to observed variations within subducting slabs. However, if more realistic plate models with subduction are employed, such that the seismic constraint is satisfied, then (1) with a $\beta \approx 1 / 3$ is more likely to be the value appropriate for studying the Earth's evolution.

\section{Numerical Models}

The numerical models presented here will illustrate that a simple exponential dependence of viscosity on temperature will lead to stagnant lids which inhibit the efficient transfer of heat; if the surface is free to advect, even though it is "stiff", then subduction occurs and the system cools as efficiently as a constant viscosity system.

then subduction occurs and the system cools as efficiently as a constant viscosity system.

All of the convection models (Table 1 ) have been solved with finite elements and have the following common teatures:

TABLE 1. Summary of Models

\begin{tabular}{rrrrlrl}
\hline case $\frac{\mathrm{Ra}_{\Omega}}{\mathrm{Rac}_{\mathrm{c}}}$ & $\begin{array}{c}\eta(\mathrm{T}) \\
\mathrm{C}\end{array}$ & $\begin{array}{c}\text { weak } \\
\text { zones }\end{array}$ & & $\mathrm{N}$ & $\mathrm{Nu}$ & $\mathrm{Ra}$ \\
& & & & & & \\
\hline a & 100 & - & no & 0.500 & 9.67 & $7.79 \times 10^{4}$ \\
b & 100 & -6.91 & no & 0.732 & 6.18 & $3.87 \times 10^{5}$ \\
c & 100 & -6.91 & yes & 0.532 & 10.68 & $9.72 \times 10^{4}$ \\
d & 100 & -9.21 & no & 0.771 & 4.93 & $9.48 \times 10^{5}$ \\
e & 500 & -6.91 & yes & 0.538 & 16.57 & $5.07 \times 10^{5}$ \\
f & $10^{3}$ & -6.91 & yes & 0.516 & 20.15 & $8.68 \times 10^{5}$ \\
\hline
\end{tabular}


a

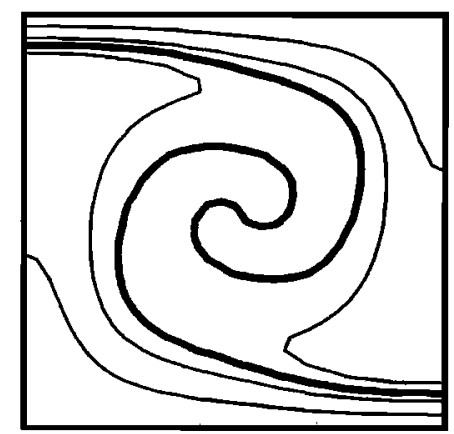

b

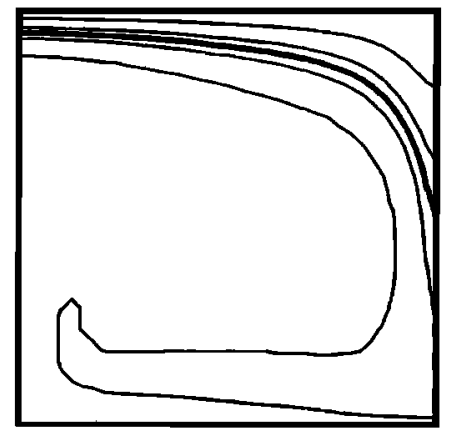

C

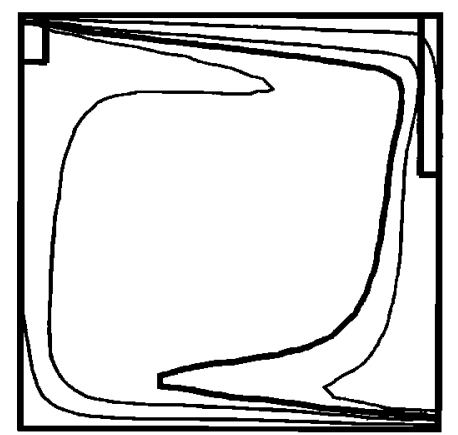

Fig. 1. Isotherms for three cases discussed in the text; the contour values are: $0.2,0.4,0.5$ (heavy contour), 0.6 , and 0.8 . a.) Case $a$ with constant viscosity, b.) Case $b$ with simple temperature dependent viscosity, and c.) Case $c$ with temperature dependent viscosity and weak zones, as indicated by the boxes in the upper corners.

flow confined to two dimensions, unit aspect ratio, free slip boundaries, and isothermal top and bottom boundaries. The FE code solves the momentum equation with a penalty function formulation and the energy equation with a streamline upwind, Petrov-Galerkin method.

Case $a$ is constant viscosity Rayleigh-Benard convection with an isothermal interior at the mean temperature $T_{b}-\Delta T / 2$ (Fig. 1a). The Rayleigh number, $R_{1} / 2$, is $100 R_{c}$, where $R_{c}(=$ $779.27)$ is the critical Rayleigh number. The surface velocity continuously varies across the top (Fig. 2a) and is unlike oceanic plates which have spatially uniform velocity. However, the fluid which cools in the top boundary layer is advected into the interior of the box and cools the interior and this is analogous to the subduction into the mantle of cold oceanic lithosphere.

Simple temperature dependent viscosity radically changes the style of convection [cf. Torrence and Turcotte, 1971]. Case $b$ has incorporated the following viscosity law

$$
\eta(T)=\eta_{0} \exp [-C(T-\Delta T / 2)]
$$

which is similar to the form used by Christensen [1984]. Case $b$ has $C=6.9$, such that the ratio of the viscosity on the top to the viscosity on the bottom is $10^{3}$. As discussed by Christensen [1984], there are some important differences between cases $a$ and $b$. First, most of the change in temperature now occurs across the top of the box, the $\Delta T / 2$ contour (heavy contour in Fig. 1b) occurs at mid-depth in this boundary layer. This layer has a high viscosity and the average surface velocity has been reduced by nearly $80 \%$ from case $a$ (Fig. 2a and Table 1). The Nusselt number has been reduced by $65 \%$ while the average temperature has been increased by $50 \%$ from case $a$. Christensen [1984] argues that since the spatially averaged temperature, $\mathrm{T}$, is significantly higher than

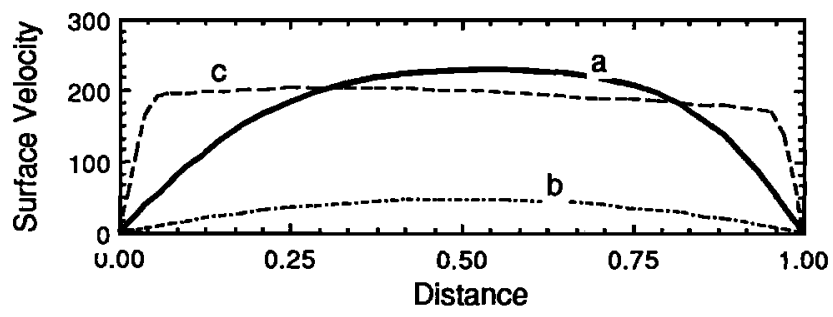

Fig. 2. Horizontal velocity across the top of the convecting region for cases $a$ (heavy solid line), $b$ (dot-dash line), and $c$ (dashed line).
$T_{b}-\Delta T / 2$, that $R_{1 / 2}$ is no longer the appropriate Rayleigh number of the system and defines a new Rayleigh number based on the viscosity at the average temperature, e.g.

$$
\operatorname{Ra}_{\mathrm{T}}=\frac{\mathrm{g} \rho \alpha \Delta \mathrm{TD}^{3}}{k \eta(\overline{\mathrm{T}})}
$$

The Rayleigh number is increased using this new definition and in "Nu-Ra" space, case $b$ is significantly shifted from case a (in Figure 3 case $b$ is labelled "Stagnant Lid"). However, like case $a$, case $b$ has a non-uniform velocity distribution across the top (Fig. 2b). The most significant difference of case $b$ from the Earth, however, is the lack of subduction, i.e. the cold fluid comprising the top boundary is not advected into the interior.

Freely moving surface plates and subducted slabs can easily be generated by introducing weak zones of viscosity $\eta_{w}$ in the top two corners [Jacoby and Schmeling, 1982]. Although highly idealized, such weak zones occur in the Earth along plate margins by way of weak faults zones. Over the last few years the weak fault zone idea, which was initially proposed from the absence of heat flow anomalies along continental transform faults [Lachenbruch and Sass, 1988], has been getting support from the observed low stresses in the Cajon Pass drill hole along the San Andreas fault [Healy and Zoback,

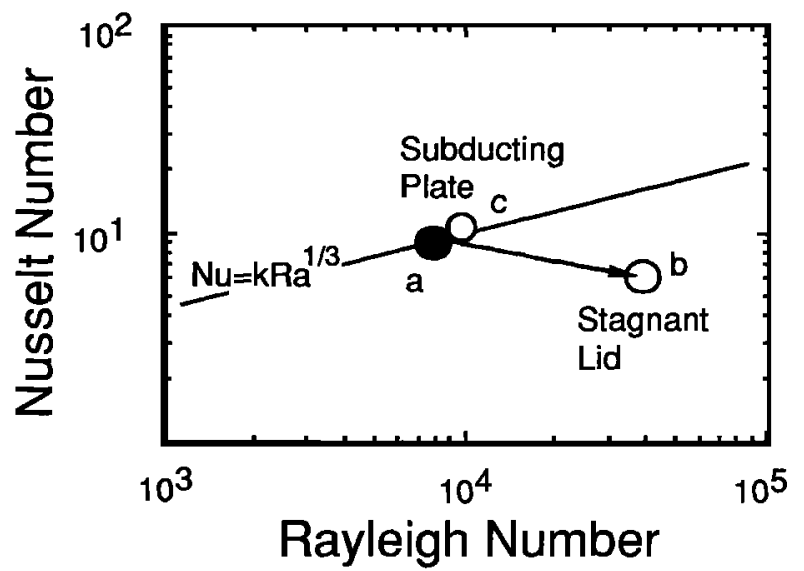

Fig. 3. Rayleigh number - Nusselt number relationship for cases $a$ (Rayleigh-Benard convection), $b$ (stagnant lid convection), and $c$ (subducting plate convection). 

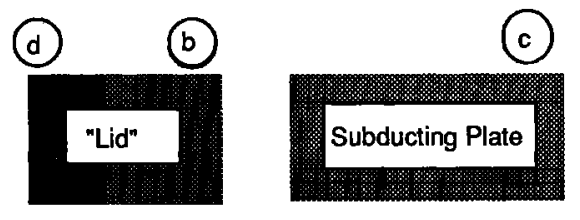

N. W. Pacific
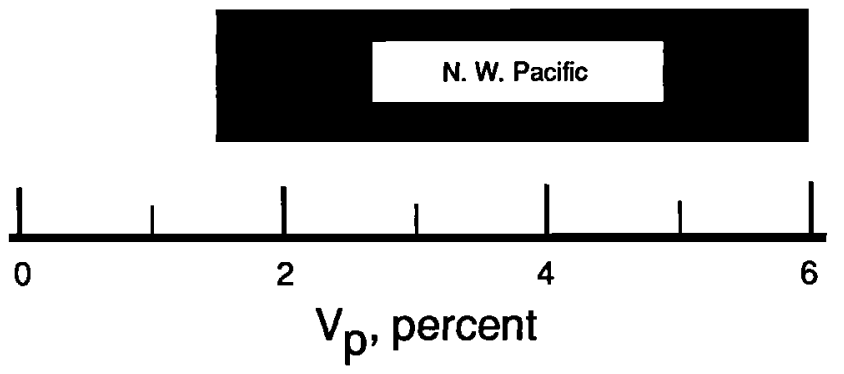

Fig. 4. Seismic compressional wave ( $p$-wave) velocity anomalies within the descending tongue of cold fluid in the convection experiments for cases $b, c$, and $d$ compared with Western Pacific slabs from Zhou and Clayton [1988].

1988]. For case $c$ the same temperature dependence is used as in case $b$, but with weak zones as is indicated in Fig. 2c. The viscosity of the weak zones, $\eta_{w}=0.05$, is somewhat higher than the minimum in viscosity $\left(\eta\left(T_{b}\right)=0.032\right)$. With such parameters the interior cools down compared to case $b$. This case satisfies the two important features of the oceanic lithosphere: horizontally uniform velocity and subduction. The average temperature is increased somewhat from case $a$ (by $6 \%$ ) and the Nusselt number increased by $10 \%$. These changes only cause a minor shift in "Nu-Ra" space (Fig. 3 where case $c$ is labelled "Subducting Plate") compared to case $a$, and this small shift is virtually along the $\mathrm{Nu} \propto \mathrm{Ra}^{1 / 3}$ curve.

As indicated above, the "stagnant lid" convection with purely temperature dependent viscosity lacks subduction, as indicated by the lack of a significant vertical tongue of cold fluid. The intensity of subduction can be quantified by scaling the maximum temperature anomaly to a compressional wave velocity, $V_{p}$, at a depth scaled by the thermal thickness of the top boundary layer. The following values were used for the scalings: a lithospheric thickness of $100 \mathrm{~km}$, a temperature difference of $1400^{\circ} \mathrm{C}$ [Cazenave, 1984; Parsons and Sclater, 1977], and a $\partial V_{p} / \partial \mathrm{T}$ of -3 to $-5.2 \times 10^{-4} \mathrm{~km} \mathrm{~s}^{-1} \mathrm{~K}^{-1}$ [Anderson et al., 1968]. Such scalings were made over a depth range of 300 to $400 \mathrm{~km}$ for the stagnant lid case $b\left(V_{p}: 1.4-2.4 \%\right)$ and the subducting plate case $c(2.9-5.0 \%)$. For the stagnant lid case, the magnitude of the seismic velocity anomaly in the "slab" depends on the intensity of the temperature dependence of viscosity (e.g. $C$ in eq. 3 ). Increasing the total variation in viscosity by a factor of 10 , to $10^{4}$, reduces $V_{p}$ to $0.9-1.5 \%$. The range in velocity differences between the interior of the slab and the adjacent mantle for the western Pacific from the Marianas to the Kuriles [Zhou and Clayton, 1988] is between 1.8-6.0\%. The "stagnant lid" convection does not generate large enough seismic velocity variations in the upper mantle, while the more realistic plate models can easily generate the required variations in $V_{p}$ (Figure 4).

Both the ability to generate plate like behavior and sufficiently fast $V_{p}$ in slabs justifies modelling an oceanic plate with weak zones on the margins. Two other cases with $\eta(T)$ and weak zones were computed at higher Rayleigh numbers in order to calculate a $\beta$ in the $\mathrm{Nu}-\mathrm{Ra}$ relation of eq. 1 . A $\beta$ of 0.3 was found for these three cases $(c, e$, and $f)$; this value is close to the $1 / 3$ calculated from boundary layer theory [Turcotte and Schubert, 1982] and the 0.319 calculated numerically for constant viscosity systems [Schubert and Anderson, 1985].
This $\beta$, however, is distinctly larger than 0.1 inferred by Christensen [1984, 1985].

\section{Conclusions}

Dynamical models have been formulated in which the style of terrestrial tectonics is better reproduced in comparison to past studies [Christensen, 1984; 1985]. The new models have plates with horizontally uniform velocity and subduction; the models of Christensen [1984] had smoothly varying velocity and no subduction. Models with subducting plates have a strong buffering between internal temperature and heat loss. The Nusselt-Rayleigh number relation for this type convection is virtually identical to constant viscosity Rayleigh-Benard convection. Past studies of parameterized convection which incorporated parameters indicative of a strong buffering between internal temperature and total heat output still provide the most physically plausible representation of the Earth's thermal evolution.

Acknowledgements. Henry Pollack provided helpful comments on this ms. Computing carried out at on the facilities of the San Diego Supercomputer Center.

\section{References}

Anderson, O.L., E. Schreiber, R. Lieberman, and N. Saga, Some elastic constant data on minerals relevant to geophysics, Rev. Geophys., 6, 491-524, 1968.

Cazenave, A., Thermal cooling of the oceanic lithosphere: New constraints from geoid height data, Earth Planet. Sci. Lett., 70, 395-406, 1984.

Christensen, U. R., Heat transported by variable viscosity convection and implications for the Earth's thermal evolution, Phys. Earth Planet. Int., 35, 264-282, 1984.

Christensen, U. R., Thermal evolution models for the Earth, J. Geophys. Res., 90, 2995-3007, 1985.

Davies, G. F., Thermal histories of convective Earth models and constraints on radiogenic heat production in the Earth, J. Geophys. Res., 85, 2517-2530, 1980.

Healy, J. H. and M. D. Zoback, Hydraulic fracturing in situ stress measurements to $2.1 \mathrm{~km}$ depth at Cajon Pass, California, Geophys. Res. Lett., 15, 1005-1008, 1988.

Jacoby, W. R. and H. Schemeling, On the effects of the lithosphere on mantle convection and evolution, Phys. Earth Planet. Int., 29, 305-319, 1982.

Jackson, M. J. and H. N. Pollack, On the sensitivity of parameterized convection to the rate of decay of internal heat sources, J. Geophys. Res., 89, 10103-10108, 1984.

Lachenbruch, A. H. and J. H. Sass, The stress heat-flow paradox and thermal results from Cajon Pass, Geophys. Res. Lett., 15, 981-984, 1988.

Parsons, B. and J. G. Sclater, An analysis of the variation of ocean bathymetry and heat flow with age, J. Geophys. Res., 82, 803-827, 1977.

Schubert, G. and C. A. Anderson, Finite element calculations of very high Rayleigh number thermal convection, Geophys. J. R. astr. Soc., 80, 575-602, 1985.

Schubert, G., D. Stevenson, and P. Cassen, Whole planet cooling and the radiogenic heat source contents of the Earth and moon, J. Geophys. Res., 85, 2531-2538, 1980.

Turcotte, D.L., F. A. Cook, and R. J. Willeman, Parameterized convection within the moon and the terrestrial planets, Proc. Lunar Planet. Sci. Con. , 10, 2375-2538, 1979.

Turcotte, D. L. and G. Schubert, Geodynamics: Applications of Continuum Mechanics to Geological Problems, 450 pp., John Wiley, New York, 1982. 
Torrance, K.E. and D.L. Turcotte, Thermal convection with large viscosity variations, J. Fluid. Mech., 47, 113-125, 1971.

Zhou, H.-W. and R. W. Clayton, P and S wave travel-time inversions for subducting slabs under island arcs of the northwest Pacific, submitted to J.Geophys. Res., 1988.
M. Gurnis, Department of Geological Sciences, 1006 C.C. Little Bldg., The University of Michigan, Ann Arbor, MI, 48109-1063.

(Received November 23, 1988; accepted December 19, 1988.) 